\title{
Comparison of the Effect of Letrozole Alone With Letrozole Plus N-Acetylcysteine on Pregnancy Rate in Patients With Polycystic Ovarian Syndrome: A Randomized Clinical Trial
}

\author{
Batool Teimouri $^{\circledR}$, Sedigheh Mollashahi ${ }^{2}$, Mahboubeh Paracheh², Farahnaz Farzaneh ${ }^{3 *}$
}

\begin{abstract}
Objectives: Different drugs are prescribed for the induction of ovulation in patients with polycystic ovary syndrome (PCOS). The aim of this study was to compare the effect of letrozole alone with letrozole plus $\mathrm{N}$-acetyl cysteine (NAC) on the pregnancy rate in patients with PCOS.

Materials and Methods: This clinical trial studyincluded317 patients with PCOS in the infertility clinic of Zahedan in 2018. Patients were randomly divided into letrozole and NAC plus letrozole groups. Then, several parameters were evaluated in both groups, including the pregnancy rate, number of follicles larger than $18 \mathrm{~mm}$, endometrial thickness, and the endometrial pattern. Finally, data were analyzed using SPSS 25, and t-test and chi-square tests were used for statistical analysis.

Results: The mean age of study participants was $28.5 \pm 4.9$ years old. The mean duration of infertility in the intervention group was 4.5 \pm 3.8 years. The mean anti-Müllerian hormone and the mean endometrial thickness of patients in the intervention group were $4.4 \pm$ $3.6 \mathrm{ng} / \mathrm{mL}$ and $7.6 \pm 2.5 \mathrm{~mm}$, respectively. Eventually, the pregnancy rate was $14.6 \%(\mathrm{n}=23)$ and $7.5 \%(\mathrm{n}=12)$ in the intervention and control groups, respectively $(P=0.046)$.

Conclusions: The results of this search revealed that the number of follicles with the size of $18 \mathrm{~mm}$ in the control group was higher compared to the intervention group, but the fertility rate in patients with PCOS receiving letrozole plus $\mathrm{N}$-acetylcysteine was significantly higher.

Keywords: Letrozole, N-acetylcysteine, Polycystic ovarian syndrome, Pregnancy
\end{abstract}

\section{Introduction}

Infertility is characterized by the inability to obtain a successful pregnancy after 6 months or more with unprotected and regular intercourse. In developing countries, the incidence of infertility is $2 \%$. The causes of infertility could be a male or female factor or a mixed factor. About 2, 40, and 2\% of infertility problems are related to a male, female, and mixed factor, respectively (13 ). The most common female cause is ovulatory disorders (40-35\%). Polycystic ovary syndrome (PCOS) is the most common cause of ovulation disorders (4).

According to the World Health Organization classification, endocrine dysfunction is the second most common functional disorder in women of childbearing age that includes hyperandrogenism and ovulation disorder. Many patients with PCOS (50\%-75\%) develop insulin resistance. These patients suffer from infertility, which is mainly due to the lack of ovulation $(5,6)$.

Additionally, PCOS is the most common endocrine disorder in women and the most common cause of infertility due to oligo ovulation or anovulation. In addition, it affects $5-10 \%$ of women of reproductive age according to the National Institutes of Health/National Institute of Child Health and Human Development criteria. According to the latest definition in 2003 (Rotterdam Criterion), PCOS has at least two criteria of three quantitative criteria, including the amount of menstrual bleeding (oligo menorrhea or amenorrhea), the clinical or biochemical symptoms of increased male hormones and sonographic findings suggesting polycystic ovary.

Furthermore, fertility problems such as infertility, bleeding caused by dysfunction, endometrial carcinoma, and postmenopausal menopause are more common in patients with PCOS depression, low self-esteem, anxiety, and metabolic disorders such as obesity, impaired glucose tolerance, type 2 diabetes, lipid disorders, and hypertension suffer (7-9). 
Key Messages

- Previous evidence demonstrates the effect of NAC on reducing semen viscosity and even as a supplement and its positive effects. Considering the fact that a significant percentage of infertility is due to hyperandrogenism and anovulation, the usefulness of this drug considering its availability and lesser side effects will be effective.

The incidence of PCOS varies between 2.2 and $26 \%$ in different countries. The reason for the difference in the prevalence of PCOS is because the clinical characteristics and biochemical characteristics of these patients may differ according to race and ethnicity (6). Moreover, the prevalence of PCOS may differ with age and community. The incidence of PCOS seems to be higher in young women compared to those over 35 years of age $(5,10,11)$.

PCOS is considered to be a complex trait arising from the interaction of genetic and environmental factors, which is usually first presented when mature gonadotropin levels are achieved at puberty. It occurs naturally in nonhuman primates and humans. The pathogenesis of PCOS can be envisioned according to a "two-hit" hypothesis, whereby the disorder arises as a congenitally programmed predisposition (first hit) that manifests in the presence of a provocative factor (second hit).

The congenital factors can be either hereditary (genetic) or acquired (e.g., maternal drugs or nutritional disorders affecting the fetus). The postnatal provocative factor is typically insulin-resistant hyperinsulinism, which may have been congenitally programmed and/or acquired postnatally due to simple (exogenous) obesity.

These complex interactions generally mimic an autosomal dominant pattern of inheritance with variable penetrance. The heritability of PCOS has been estimated at over $70 \%$ based on studies in identical twin sisters (10).

Currently, the first applied drug for induction ovulation in patients with the endocrine syndrome is estrogen receptor modulator clomiphene citrate as an estrogen receptor modulator, and its success rate has been reported in various cases. Clomiphene citrate resistance is found in $15 \%-20 \%$ of patients that ovulation does not persist after standard drug therapy, therefore, finding strategies to increase the success of induction ovulation in infertile patients has great importance.

Other proposed drugs for the induction of ovulation in patients with PCOS may include aromatase inhibitors, including letrozole, which, like clomiphene reduces the hypothalamic-pituitary-axis estrogenic stimulation by decreases estrogen biosynthesis. One of the benefits of letrozole is the lower probability of multiple pregnancies and greater endometrial thickness at the end of the treatment. It also has a half-life of about 45 hours and has less adverse effects on estrogen target tissues such as the endometrium and cervix compared to clomiphene (10).
Letrozole as the first-line treatment for ovulation induction can be as effective as clomiphene. In some studies, letrozole has a success rate of $70 \%-84 \%$ in ovulation induction, $20 \%-27 \%$ pregnancy rate, and endometrial thickness after treatment with letrozole has been reported 7-9 $\mathrm{mm}(5,10)$.

Other studies have shown that insulin sensitivity enhancers decrease the levels of hyperandrogenism and hypertension in patients with PCOS and are effective in induction ovulation in these patients $(10,12)$.

Another suggested drug for the induction of ovulation is $\mathrm{N}$-acetyl cysteine (NAC). It is an acetylated derivative of L-cysteine and is safe, inexpensive, and affordable. In addition, it is a great source of sulfhydryl groups and converts into metabolites that stimulate glutathione production and increase detoxification and directly decompose free reactive oxygen species (12).

NAC also has an effect on insulin release in beta cells in the pancreas and regulates insulin receptors on human erythrocytes (13). Effects such as lowering serum androgen levels and improving homocysteine status and lipid profile have also been reported in patients with PCOS (14).

Previous evidence demonstrates the effect of NAC on reducing semen viscosity and even as a supplement and its positive effects. Considering the fact that a significant percentage of infertility is due to hyperandrogenism and anovulation, the usefulness of this drug considering its availability and lesser side effects will be effective.

Therefore, this study was performed to investigate the inducible effect of NAC on letrozole in ovulation induction in infertile women with PCOS.

\section{Materials and Methods}

This clinical trial study was performed on 317 patients with PCOS referring to the infertility clinic of Zahedan in 2018. Patients were selected based on two criteria of the three main criteria (oligo/anovulation), clinical or laboratory evidence of hyperandrogenism, polycystic ovary morphology on ultrasound (Rotterdam criteria), normality of thyroid function and prolactin, normal semen analysis, at least one year of infertility, and the existence of at least one patient tube. On the other hand, the exclusion criteria were thyroid dysfunction and prolactin, tubal or uterine disorders leading to infertility, moderate and severe oligozoospermia, asthenozoospermia, and teratozoospermia, size of an ovarian cyst greater than 6 $\mathrm{cm}$, history of asthma, and any drug affecting glucose metabolism.

Patients were randomly divided into control (letrozole alone) and intervention (letrozole NAC plus). It was impossible to be blinded in this study because patients did not visit simultaneously and were unaware of the treatment of others. There was virtually no placebo.

The control group was selected from patients with the same inclusion criteria and standard treatment of 
letrozole were visited by the same infertility fellowship on different days. Totally, the variables were small and no bias was possible.

The control group received $5 \mathrm{mg}$ of letrozole daily for day 5 and the intervention group received $5 \mathrm{mg}$ of letrozole $5 \mathrm{mg}$ daily with $1200 \mathrm{mg}$ NAC for 5 days. The patients' medicines were obtained from a factory.

Five days after the last dose of letrozole, patients underwent a vaginal ultrasound to measure endometrial thickness, as well as the number and diameter of mature follicles. The ultrasound of the patients was performed by one infertility fellowship using an ultrasound device. In the presence of at least one follicle equal to or greater than $18 \mathrm{~mm}, 5000$ units of human chorionic gonadotropin (HCG) were injected intramuscularly and intercourse was recommended approximately 12,36 , and 60 hours after the injection.

On the 12th day after HCG injection, serum betaHCG levels were measured to check for pregnancy. The ovulation and pregnancy rates in each group were calculated and finally compared as well.

\section{Statistical Analysis}

The central and dispersion indices were used to describe the quantitative data, and frequency distribution tables (absolute and relative) were employed to describe the qualitative data. $\mathrm{T}$ test and the nonparametric test were used to analyze quantitative data for normal and abnormal data, respectively. The data were analyzed by SPSS version 25.

\section{Results}

The mean age of patients was $28.5 \pm 4.9$ years $(\mathrm{P}=0.441)$. The mean BMI was $25.9 \pm 4.3 \mathrm{~kg} / \mathrm{m}^{2}$ and $26.6 \pm 5.7 \mathrm{~kg} /$ $\mathrm{m}^{2}$ in intervention and control groups, respectively. The mean duration of infertility in the intervention and control groups was $4.5 \pm 3.8$ years and $4.3 \pm 2.9$ years $(P=$ 0.713 ), respectively (Table 1 ).

The mean AMH was $4.4 \pm 3.6 \mathrm{ng} / \mathrm{mL}$ and $5.1 \pm 2.5 \mathrm{ng} /$ $\mathrm{mL}$ in the intervention and control groups, respectively $(P=0.200)$. In addition, $75(47.5 \%)$ follicles larger than $18 \mathrm{~mm}$ and $83(52.5 \%)$ follicles smaller than $18 \mathrm{~mm}$ were observed in the intervention group while $96(60.4 \%)$ cases had follicles larger than $18 \mathrm{~mm}$ and 63 (39.6\%) of them had follicles smaller than $18 \mathrm{~mm}$ in the control group, this difference was statistically significant $(P=0.021)$. In the present study, the pregnancy rate was 23 patients (14.6\%) in the intervention group, and $12(7.5 \%)$ cases were pregnant in the control group and this difference was statistically significant $(P=0.046)$ as well (fellowchart1). Endometrial thickness was $7.6 \pm 2.5 \mathrm{~mm}$ and $7.9 \pm 2.7$ $\mathrm{mm}$ in the intervention and control groups, respectively, representing no statistically significant difference between the two groups $(P=0.333)$. In the present study, 18 $(11.4 \%)$ and $140(88.6 \%)$ patients had a homogeneous endometrial pattern and three-line endometrial pattern in the intervention group, respectively. In the control group, 15 (9.4\%) cases had a homogenous endometrial pattern and $144(90.6 \%)$ of them had a three-layer endometrial pattern. This difference was not statistically significant $(P=0.556)$, the details of which are presented in Table 2 .

\section{Discussion}

Infertility in women with PCOS is a serious problem and there are various ways to increase their pregnancy rate. Letrozole significantly increases the probability of pregnancy in patients with the polycystic ovary, but the success rate in this procedure is not precisely known and reaches $20 \%$ in the best cases (15). On the other hand, Kilic-Okman and Kucuk described NAC as an effective drug in lowering serum insulin and testosterone while improving the serum homocysteine level and lipid profile in PCOS patients (14). Pregnancy rates were assessed in patients with PCOS. This study evaluated the effect of NAC on letrozole for pregnancy rate in patients with PCOS.

The results of our study showed that the pregnancy rate was $14.6 \%$ and $7.5 \%$ in intervention and control groups, respectively, and follicles larger than $18 \mathrm{~mm}$ were observed in the intervention (47.5\%) and control (60.4\%) groups, showing the significant effect of NAC on increasing fertility in these patients (Figure 1).

In their study, Mostajeran et al divided 130 PCOS patients into letrozole plus NAC and letrozole alone and represented that ovulation and pregnancy rates were significantly higher in the letrozole plus NAC group, which is consistent with our study, but unlike our study, the number of follicles greater than $18 \mathrm{~mm}$ was also significant in the letrozole plus NAC group (15).

In line with the findings of our study, Holzer et al found that the success rate of letrozole in ovulation induction was $70 \%-84 \%$, and the pregnancy rates of $20 \%-27 \%$ were observed and finally, endometrial thickness was between

Table 1. Comparison of Primary Variables in the 2 Study Groups

\begin{tabular}{lccc}
\hline \multirow{2}{*}{ Variable } & \multicolumn{2}{c}{ Group } & \\
\cline { 2 - 3 } & $\begin{array}{c}\text { Letrozole + NAC } \\
(\mathbf{n = 1 5 8 )}\end{array}$ & $\begin{array}{c}\text { Letrozole } \\
(\mathbf{n = 1 5 9 )}\end{array}$ & $\boldsymbol{P}$ Value \\
\hline Age & $28.2 \pm 5.0$ & $28.7 \pm 4.8$ & 0.441 \\
BMI & $25.9 \pm 4.3$ & $26.6 \pm 5.7$ & 0.251 \\
Duration of infertility & $4.5 \pm 3.8$ & $4.3 \pm 2.9$ & 0.713 \\
\hline
\end{tabular}

Note. BMI, Body mass index; NAC, N-acetyl cysteine.

Table 2. Comparison of Studied Variables Between the 2 Groups

\begin{tabular}{llll}
\hline \multirow{2}{*}{ Variable } & \multicolumn{2}{l}{ Group } & \\
\cline { 2 - 3 } & $\begin{array}{l}\text { Letrozole + NAC } \\
(\mathbf{n = 1 5 8})\end{array}$ & $\begin{array}{l}\text { Letrozole } \\
(\mathbf{n = 1 5 9 )}\end{array}$ & $\boldsymbol{P}$ Value \\
\hline AMH & $4.4 \pm 3.6$ & $5.1 \pm 5.2$ & 0.200 \\
Follicle $>18 \mathrm{~mm}$ & $75(47.5 \%)$ & $96(60.4 \%)$ & 0.021 \\
Pregnancy rate & $23(14.6 \%)$ & $12(7.5 \%)$ & 0.046 \\
Endometrial thickness $(\mathrm{mm})$ & $7.6 \pm 2.5$ & $7.9 \pm 2.7$ & 0.333 \\
\hline
\end{tabular}

Note. AMH, Anti-Müllerian hormone; NAC, N-acetyl cysteine. 


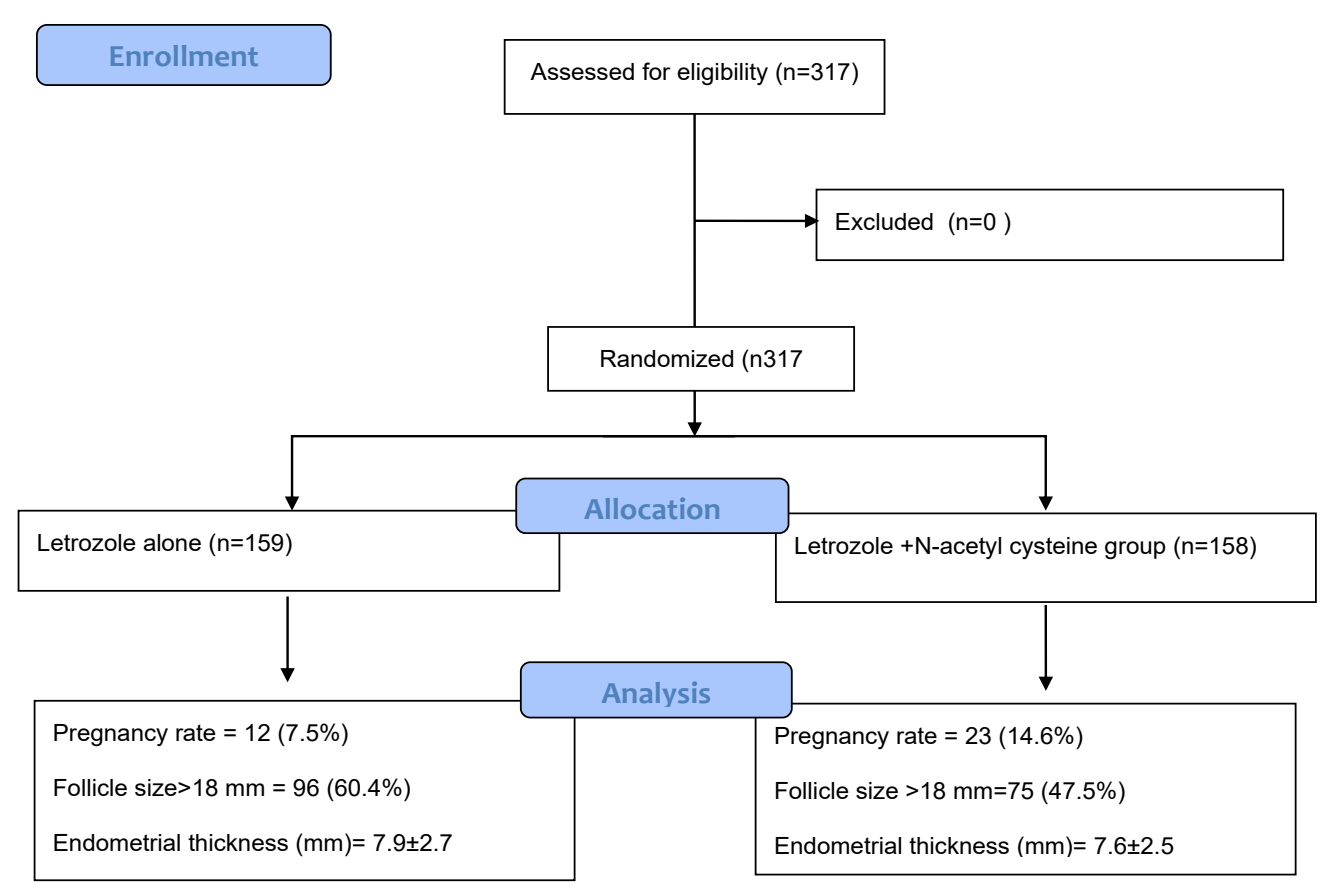

Figure 1. Comparison of Pregnancy Rate and Follicle Size Between the Two Groups.

7 and $9 \mathrm{~mm}(10)$.

Based on the results of a study by Dabbaghi Ghale et al on 40 women with PCOS, NAC failed to increase the success rate of letrozole in induction ovulation in these women, and the pregnancy rate was observed in $15 \%$ and $20 \%$ of the control and intervention groups, respectively. Similar to our study, the fertility rate was higher in the intervention group and was statistically significant. Furthermore, the mean endometrial thickness was similar to our study in the two groups although the mean number of follicles in the intervention group was higher in comparison with the control group (16).

Other studies also used clomiphene and NAC. For example, Riyahi Nezhad et al reported that adding NAC to clomiphene significantly increased the follicle size and pregnancy rate in women (17). In a similar study by Salehpour et al, 167 infertile women with PCOS were randomly treated with clomiphene, NAC plus clomiphene, and placebo (17)and the results revealed that ovulation and pregnancy rates were significantly higher in the clomiphene plus NAC groups compared to the clomiphene and placebo groups (18).

In the study of Badawy et al, ovulation rate and endometrial thickness were reported after a cycle of treatment with clomiphene and NAC significantly higher than before. The observed mean follicles after NAC were also significantly higher (19).

Other studies compared the effect of clomiphene and NAC with clomiphene alone for the induction of ovulation in patients with unexplained infertility. Patients were compared in two groups receiving clomiphene alone and clomiphene with NAC. Based on their findings, ovulation rate and endometrial thickness were significantly higher than before with a clomiphene and NAC cycle. Finally, the mean follicles observed after NAC were significantly higher $(20,21)$.

\section{Conclusions}

The findings of our study and other studies showed that the addition of NAC to letrozole, similar to clomiphene, increases the fertility rate in women with PCOS and promotes the success of infertility.

\section{Study Limitations}

The optimal therapeutic effects of NAC may be limited to a specific range of patients, therefore, it is recommended to conduct studies on patients with high BMI, insulinresistant, or clomiphene-resistant patients although it is not feasible due to the limited cost of university approval. Eventually, studies with higher doses of NAC or longer evaluations may give more hope for the efficacy of this drug in ovulation, which requires further studies.

\section{Authors' Contribution}

BT and FF conceived of the presented idea, developed the theory, and performed the examination. In addition, SM and MP verified analytical methods and supervised the findings of this work, and then discussed the results and contributed to the final manuscript, and finally, FF wrote the article.

\section{Conflict of Interests}

Authors declare that they have no conflict of interests.

\section{Ethical Issues}

This research is a dissertation approved by the Ethics Committee of the Zahedan University of Medical Sciences in 2018 (Ethics 
Code: IR.ZAUMS.REC.1397.167) Additionally, the study was registered at Iranian Registry of Clinical Trials (identifier: IRCT20180425039418N2; https://www.irct.ir/trial/33932).

\section{Financial Support}

The authors declare that the current research was done with no supports from any organizations and/or universities.

\section{Acknowledgments}

Special thanks go to all the distinguished professors, colleagues, and patients who collaborated in the implementation of this project.

\section{References}

1. Behnoud N, Bahrami R, Kordafshari G, Farzaneh F, Mohammadi Kenari $\mathrm{H}$. Management of early menopause using traditional Persian medicine: a case report. Int J Womens Health Reprod Sci. 2019;7(2):231-236. doi:10.15296/ijwhr.2019.39

2. Behnoud N, Farzaneh F, Ershadi S. The effect of clomiphene citrate versus letrozole on pregnancy rate in women with polycystic ovary syndrome: a randomized clinical trial. Crescent J Med Biol Sci. 2019;6(3):335-340.

3. Bayer S. The Boston IVF Handbook of Infertility: A Practical Guide for Practitioners Who Care for Infertile Couples. CRC Press; 2017.

4. Abtahi-Eivari SH, Moghimian M, Soltani M, et al. The effect of Galega officinalis on hormonal and metabolic profile in a rat model of polycystic ovary syndrome. Int J Womens Health Reprod Sci. 2018;6(3):276-282. doi:10.15296/ijwhr.2018.46

5. Fritz MA, Speroff L. Clinical Gynecologic Endocrinology and Infertility. Lippincott Williams \& Wilkins; 2012.

6. Maino Vieytes C. Polycystic Ovarian Syndrome Nutritional Management. Military Families Learning Network; 2019.

7. Wang ET, Calderon-Margalit R, Cedars MI, et al. Polycystic ovary syndrome and risk for long-term diabetes and dyslipidemia. Obstet Gynecol. 2011;117(1):6-13. doi:10.1097/AOG.0b013e31820209bb

8. Xiang SK, Hua F, Tang Y, Jiang XH, Zhuang Q, Qian FJ. Relationship between serum lipoprotein ratios and insulin resistance in polycystic ovary syndrome. Int J Endocrinol. 2012;2012:173281. doi:10.1155/2012/173281

9. Ouladsahebmadarek E, Khaki A. Ultra-structural study by transmission electron microscopy: effect of omega-3 on ovary cell organelles after experimental induced poly cystic ovary syndrome. Int J Womens Health Reprod Sci. 2014;2(3):186194. doi:10.15296/ijwhr.2014.27

10. Holzer $\mathrm{H}$, Casper $\mathrm{R}$, Tulandi T. A new era in ovulation induction. Fertil Steril. 2006;85(2):277-284. doi:10.1016/j. fertnstert.2005.05.078

11. Saei Ghare Naz M, Ramezani Tehrani F, Alavi Majd H, et al. The prevalence of polycystic ovary syndrome in adolescents: a systematic review and meta-analysis. Int J Reprod Biomed. 2019;17(8):533-542. doi:10.18502/ijrm.v17i8.4818

12. Saha L, Kaur S, Saha PK. N-acetyl cysteine in clomiphene citrate resistant polycystic ovary syndrome: a review of reported outcomes. J Pharmacol Pharmacother. 2013;4(3):187-191. doi:10.4103/0976-500x.114597

13. Santini MT, Cametti C, Indovina PL, Peterson SW. Menadione induces changes in the membrane electrical properties associated with downregulation of insulin receptors in human erythrocytes. Exp Hematol. 1998;26(6):466-471.

14. Kilic-Okman T, Kucuk M. N-acetyl cysteine treatment for polycystic ovary syndrome. Int J Gynaecol Obstet. 2004;85(3):296-297. doi:10.1016/j.ijgo.2004.03.002

15. Mostajeran F, Ghasemi Tehrani H, Rahbary B. N-acetylcysteine as an adjuvant to letrozole for induction of ovulation in infertile patients with polycystic ovary syndrome. Adv Biomed Res. 2018;7:100. doi:10.4103/abr.abr_157_17

16. Dabbaghi Ghale T, Mohebbi F, Movahed F, Pakniat H, Yazdi Z. Effect of $\mathrm{N}$-acetylcysteine with letrozole to induction of ovulation in infertile women with polycystic ovarian syndrome. Journal of Gorgan University of Medical Sciences. 2016;18(3):1-6. [Persian].

17. Riyahinezhad S, Ghasemi Tehrani H, Movahedi M, Soltani N, Haghighat $\mathrm{F}$. The effectiveness of using of $\mathrm{N}$ acetyl cysteine together with clomiphene citrate on ovulation indication in the patients with polycystic ovary syndrome referred to Isfahan reproduction and infertility center, 2013. J Sci Res Dev. 2015;2:210-214.

18. Salehpour S, Akbari Sene A, Saharkhiz N, Sohrabi MR, Moghimian F. N-acetyl cysteine as an adjuvant to clomiphene citrate for successful induction of ovulation in infertile patients with polycystic ovary syndrome. J Obstet Gynaecol Res. 2012;38(9):1182-1186. doi:10.1111/j.14470756.2012.01844.x

19. Badawy A, State O, Abdelgawad S. N-acetyl cysteine and clomiphene citrate for induction of ovulation in polycystic ovary syndrome: a cross-over trial. Acta Obstet Gynecol Scand. 2007;86(2):218-222. doi:10.1080/00016340601090337

20. Shoorei H, Banimohammad M, Kebria MM, et al. Hesperidin improves the follicular development in 3D culture of isolated preantral ovarian follicles of mice. Exp Biol Med (Maywood). 2019;244(5):352-361. doi:10.1177/1535370219831615

21. Khodaeifar F, Fazljou SMB, Khaki A, et al. Investigating the role of hydroalcoholic extract of Apium graveolens and Cinnamon zeylanicum on metabolically change and ovarian oxidative injury in a rat model of polycystic ovary syndrome. Int J Womens Health Reprod Sci. 2019;7(1):92-98. doi:10.15296/ ijwhr.2019.15

(C) 2021 The Author(s); This is an open-access article distributed under the terms of the Creative Commons Attribution License (http:// creativecommons.org/licenses/by/4.0), which permits unrestricted use, distribution, and reproduction in any medium, provided the original work is properly cited. 